\title{
Surgical Treatment of Objective Patellar Instability: Long-Term Results
}

\author{
Massimo Berruto $^{1}$ Francesco Mattia Uboldi $^{2}$ Paolo Ferrua $^{1}$ Giovanni Vergottini ${ }^{3}$ Andrea Manunta ${ }^{2}$
}

1 Department of Knee Surgery, Gaetano Pini Hospital, Milan, Italy
2 Department of Orthopaedics, University of Sassari, Sassari, Italy
${ }^{3}$ Orthopaedic and Traumatology Unit, Alessandro Manzoni Hospital,
Lecco, Italy

Address for correspondence Francesco Mattia Uboldi, MD, Department of Orthopaedics, University of Sassari, Viale San Pietro 43/B, Sassari 07100, Italy (e-mail: fraubo@gmail.com).

Joints 2018;6:33-36.

\begin{abstract}
Keywords

- patellar instability

- patellofemoral

- Elmslie-Trillat

- osteoarthritis

- tibial tubercle transfer

Purpose To evaluate the long-term results of classical "à la carte" surgical treatment of objective patellar instability as proposed by Dejour in 1987.

Methods A multicentric retrospective study was conducted evaluating patients that underwent surgical procedure with a 10-year minimum follow-up (mean 12.7; range, $10-$ 15). Surgical procedures were medial transfer of the tibial tubercle transfer according to Elmslie and Trillat in 38 cases, plasty of the vastus medialis obliquus according to Insall in 15 cases, open lateral retinacular release in 13 cases, capsuloplasty in 3 cases, and trochleoplasty in 1 case. Different combinations of surgical procedures were adopted according to the pathological features. Subjective outcome was assessed with the visual analog scale (VAS), Kujala score, subjective International Knee Documentation Committee (IKDC) score, Tegner score, and Crosby and Insall scale. Radiographic exams were used to assess the patellar tilt by the Laurin's angle and patellofemoral osteoarthritis (OA) according to the Iwano radiological OA scale.

Results Forty patients were evaluated. Subjectively, $60 \%$ of patients achieved a result judged good, $34 \%$ sufficient, and $6 \%$ poor. There were only two cases of recurrence of instability. Mean score results were Kujala score $73.4 \pm 9.9$ (range, 55-95), VAS $4.5 \pm 1.2$ (range, 1-6), IKDC $64.8 \pm 7.9$ (range, 51-88), and Tegner score 4 . Only nine patients returned to sports activities). Ten patients developed a grade I patellofemoral OA, 8 patients a grade II, and 22 patients a grade III. Average patellar tilt was $10^{\circ} \pm 3.9^{\circ}$.

Conclusion This retrospective study showed that the traditional surgical procedure was successful for the treatment of patellar instability, but it did not prevent symptomatic patellofemoral OA.

Level of Evidence Level IV, retrospective case series.
\end{abstract}

\section{Introduction}

The patellofemoral (PF) joint is characterized by a low degree of congruence. Its passive stability depends on the shape of the trochlea and the patella, while its active stability is ensured by surrounding muscles that allow the patella to be actively centered into the trochlea during knee movements.

received

September 9, 2017

accepted after revision

February 4, 2018

published online

March 7, 2018
The most important anatomical factors causing patellar instability are trochlear dysplasia, exceeding distance between tibial tubercle (TT) and trochlear groove (TG), and a high riding patella (patella alta). ${ }^{1}$ Among secondary factors altering patellar stability are the following: exceeding femoral anteversion, tibial external rotational deformity, and recurved and valgus knee. An adequate selection of

Copyright @ 2018 Georg Thieme Verlag KG Stuttgart · New York
License terms

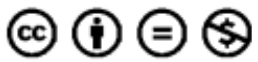


patients for eventual surgical treatment is based on discriminating among different types of patients presenting with knee pain or instability. The most suitable classification for PF pathology was proposed by Dejour et $\mathrm{al}^{2}$ and is based on three main categories. Objective patellar instability (OPI)patients belonging to this group had at least one documented episode of patellar dislocation or subluxation and at least one of the most important instability factors: trochlear dysplasia, patella alta, and pathological TT-TG distance. Potential instability-category to which belong cases where no dislocation or subluxation was reported, but at least one of the main instability factors is reported and the main symptom is pain. Patellar pain syndrome whose main symptom is pain, but no episodes of dislocation or subluxation are reported and none of the three main instability factors is reported.

This classification explains why the anterior knee pain can be secondary to but not exclusively attributable to a PF pathology. Although the occurrence of one of the three main factors can cause pain (potential instability), painful PF syndromes do not necessarily show dysplastic features.

Normally, surgery is indicated for OPI treatment. Surgical procedures aim at correcting soft tissue abnormalities (muscles and ligaments) and/or bony structures. Combined procedures can be necessary, depending on the pathological features.

The current treatment is an evolution of "à la carte menu" introduced by Dejour, ${ }^{3}$ which still aims at correcting any occurring predisposing factor. Results of these treatments are frequently reported at short-term follow-up, but no consistent literature exists on long-term outcome of this treatment approach. ${ }^{4}$

The purpose of the present study was to evaluate the longterm results of classical "à la carte" surgical treatment of OPI in terms of effectiveness on addressing patella instability and possible consequences on integrity of the PF joint. The hypothesis of the study was that surgical treatment of OPI is effective in restoring patella stability, but cannot prevent PF osteoarthritis (OA).

\section{Methods}

A multicenter retrospective study was conducted on patients who underwent a surgical treatment of OPI at minimum 10-year follow-up (mean 12.7; range, 10-15). During the examined period (1995-2005), a total of 56 patients underwent surgery for OPI in the two facilities involved in the study. We had a dropout of $29 \%$ due to the death of two patients (other causes), the untraceability of 10 patients, and denial by 4 study subjects.

Overall, 40 patients were enrolled: 28 females and 12 males with a mean age of 25.8 years (range, $16-44$ ). Surgical treatments performed (24 left knees and 16 right knees) were medial transfer of the tibial tubercle transfer (TTT) according to Trillat et $\mathrm{al}^{5}$ in 38 cases, plasty of the vastus medialis obliquus (VMO) according to Insall in 15 cases, open lateral retinacular release (LRR) in 13 cases, capsuloplasty in 3 cases, and trochleoplasty in 1 case. Different combinations of surgical procedures were adopted according to the pathological features and by following the "à la carte menu" introduced by Dejour ${ }^{3}$ (-Fig. 1). No significant differences for age and gender were observed in the population from the two facilities $(p=0.8$ and $p=0.9$ for age and gender, respectively), and according to surgical technique applied.

Subjective outcome was assessed with the visual analog scale (VAS), Kujala score, subjective International Knee Documentation Committee (IKDC) score, Tegner score, and Crosby and Insall scale. Radiographic exams were used to assess PF OA according to the Iwano radiological OA scale ${ }^{6}$ and the patellar tilt by the Laurin's angle, which is formed by

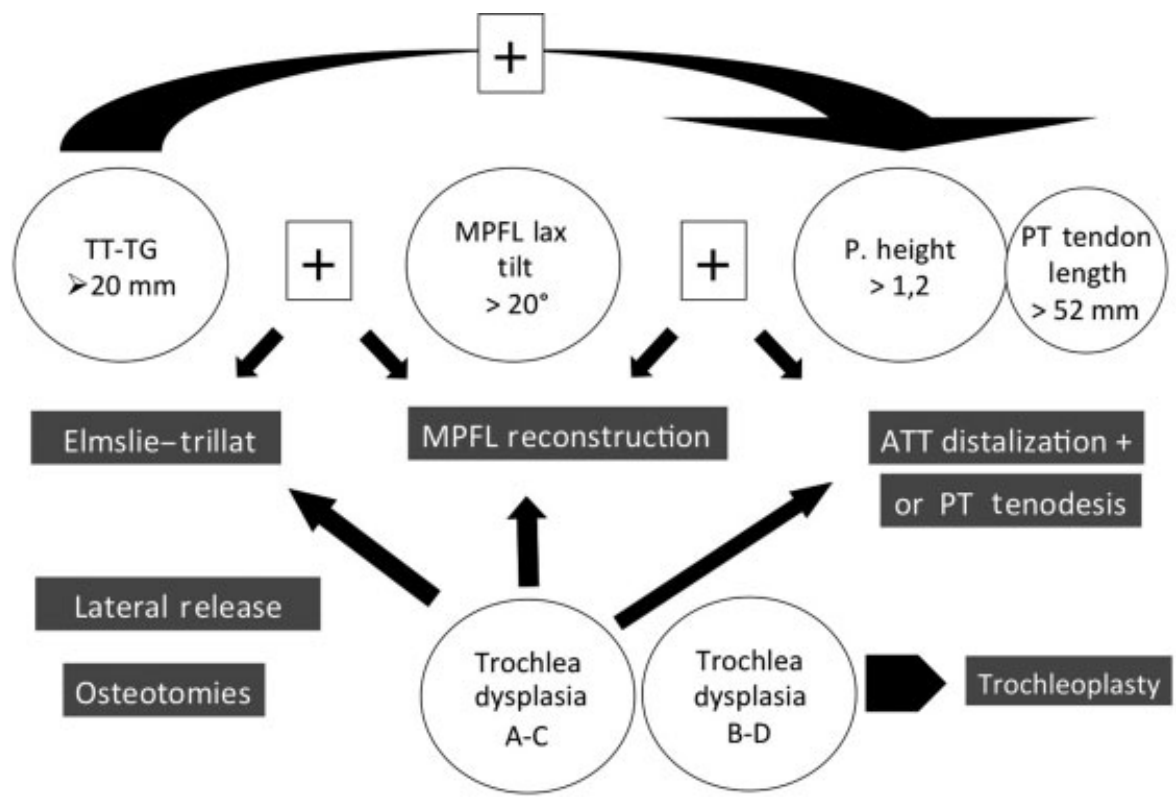

Fig. 1 Current “à la carte menu” for objective patellar instability. Abbreviations: ATT, anterior tibial tubercle; MPFL, medial patellofemoral ligament; TG, trochlear groove; TT, tibial tubercle. 
the anterior tangent to the femoral condyle and the tangent to the lateral patellar facet. ${ }^{7}$

\section{Results}

Two cases of recurrence of patellar dislocation due to trauma were reported. Mean VAS was $4.5 \pm 1.2$ (range, 1-6), mean Kujala score was $73.4 \pm 9.9$ (range, 55-95), mean subjective IKDC score was $64.8 \pm 7.9$ (range, 51-88), and mean Tegner score was $4 \pm 1.7$. Only nine patients were able to practice sports as they used to prior to the first patella dislocation. Subjective evaluation according to the Crosby and Insall scale was good in $60 \%$ of patients, sufficient in $34 \%$, and insufficient in $6 \%$.

No cases of joint stiffness were reported. Quadriceps weakness with a difference of more than $2 \mathrm{~cm}$ of thigh circumference was reported in $70 \%$ of the cases that received a plasty of VMO.

According to Iwano OA scale, a total of 10 patients developed a grade I OA, 8 patients a grade II, and 22 patients a grade III (-Fig. 2). The mean patellar tilt was $10^{\circ} \pm 3.9^{\circ}$ (range, $5^{\circ}-20^{\circ}$ ) ( - Fig. 3). No significant differences were

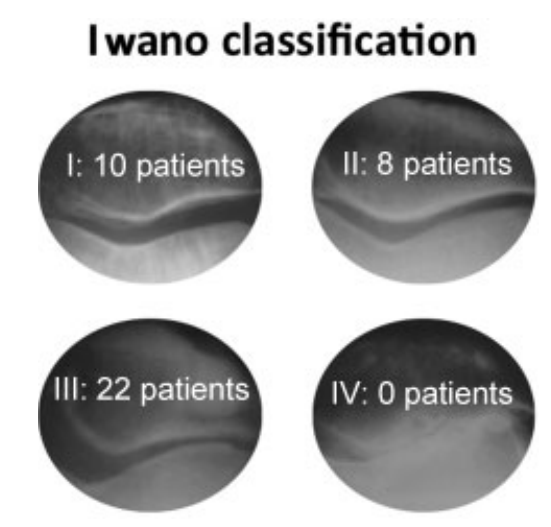

Fig. 2 Radiographic results at long-term follow-up according to Iwano classification for patellofemoral osteoarthritis. ${ }^{5}$

\section{Patellar tilt}

\section{$\alpha: 10^{\circ} \pm 3.9^{\circ}\left(\right.$ range $\left.5^{\circ}-20^{\circ}\right)$}

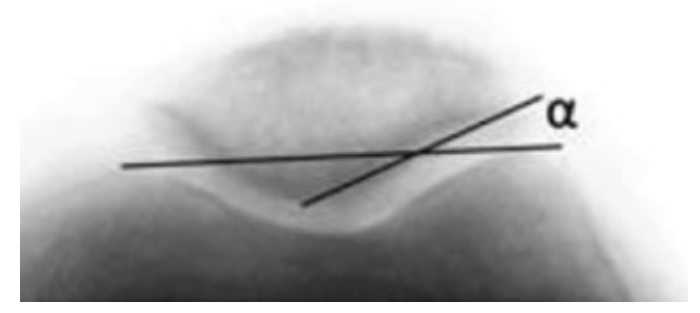

Fig. 3 Radiographic results at long-term follow-up: mean patellar tilt angle according to Laurin. ${ }^{7}$ observed between the two facilities involved in the study for all the outcome measures.

\section{Discussion}

The most important result reported in our study was the effectiveness of our surgical approach to treat patellar instability at a minimum 10-year follow-up. Indeed, the main objective in any OPI should aim at avoiding any recurrence of instability. Following surgical procedure, patients should return to normal daily activities and possibly sports activities without restrictions.

Based on the most relevant literature on this subject, the extensor mechanism realignment by medial and/or distal transposition of the TT is one of the most common and may be overused procedures in the treatment of PF syndromes. This procedure was introduced by Roux ${ }^{8}$ and proposed over the years with several modifications, including the technique described by Trillat et al. ${ }^{5}$

The recurrence rate reported after medial TTT varies between $0 \%$ up to $13 \%$. Brown et $\mathrm{al}^{9}$ reported no cases of dislocation in nine patients at a 3-year follow-up. Kumar et al ${ }^{10}$ analyzed 27 knees in 22 patients treated with Elmslie-Trillat procedure and reported good-to-optimal results in $81 \%$ of cases at an average 42-month follow-up. The authors highlighted a significant correlation between the $\mathrm{Q}$ angle and subjective results, with optimal-to-excellent results being observed with values of $\mathrm{Q}$ angle equal to or lower than $10^{\circ}$; conversely, results were less satisfactory when $\mathrm{Q}$ angle was equal to or higher than $15^{\circ}$. Other studies, even with a longer follow-up, confirm satisfactory results with the Elmslie-Trillat procedure in terms of stability, but occurrence of anterior knee pain was reported in a percentage varying between $8 \%$ an $17 \%$, also in patients asymptomatic prior to the intervention. ${ }^{11-13}$

Nakagawa et $\mathrm{al}^{11}$ reported excellent long-term results of medial TTT in terms of patellar stability with a dislocation rate of $13 \%$ at a 13 -year average follow-up (10-year minimum follow-up); however, a worsening of Fulkerson's index and occurrence of anterior chronic knee pain were reported.

Carney et al $^{12}$ analyzed a group of 18 patients at an average follow-up of 26 years who had previously been evaluated at 3-year follow-up and found similar results for both studies in terms of recurrence rate, albeit there was a functional worsening over time.

Servien et $\mathrm{al}^{14}$ analyzed 140 patients (190 knees) at an average follow-up of 60 months (range, 24-152) who underwent VMO plasty and medial TTT according to Elmslie-Trillat for the treatment of OPI. Overall, 95\% of patients were highly satisfied or satisfied; the others were dissatisfied. Recurrence rate of instability was $5.2 \%$. The most frequently reported problem by patients was kneeling (68\% of cases).

In the present study, we reported only two cases of recurrence of instability (one for each facility); however, residual anterior pain mean was frequent with a high mean VAS (4.5 points). Mean IKDC subjective score equal to 64.8 confirmed an incomplete recover of joint function, with just $25 \%$ of patients going back to sports activity at the same levels before the occurrence of instability. 
Dejour et al ${ }^{1}$ reported that $83 \%$ of patients with OPI showed a patellar tilt over $20^{\circ}$ compared with just 3\% of healthy patients. In $95 \%$ of our cases, the postoperative assessment of patellar tilt resulted within normal values. However, radiographic assessment of PF OA showed more severe degenerative changes compared with the contralateral uninjured knee. Farr et $\mathrm{al}^{15}$ reported a clear association between distal bone realignment procedures (like Elmslie-Trillat) and long-term patellar degeneration due to $\mathrm{OA}$, especially in patients with delayed treatment. Schüttler et $\mathrm{al}^{16}$ reported a significant increase in OA prevalence in patients who underwent proximal realignment (e.g., Insall procedure) compared with baseline conditions (from $10 \%$ to $43 \%$ of patients with grade II-IV $\mathrm{OA}$ according to Iwano classification).

Preoperative clinical evaluation and imaging play a key role in the planning of surgical treatment of OPI, especially for bony procedures like medial TTT. Measurement of TT-TG distance and the occurrence of trochlear dysplasia must be accurately assessed to avoid surgical treatments that may trigger medial overpressure or may lead to medial instability in more severe cases. ${ }^{1}$ Moreover, surgical correction of extensor mechanism alignment alters joint loading and contact pressure, thus increasing the risk of occurrence of late $\mathrm{OA} .{ }^{17}$ However, also delay of surgical intervention might cause recurrent dislocations and further chondral damage, with a high risk of developing early OA.

The present study has some limitations. First, surgical treatments were differently combined according to the Lyonnaise School and we could not assess the efficacy of a standard procedure. Second, the lack of preoperative evaluation limited validity of subjective outcome assessment. Finally, the sample size was small and heterogeneous for age.

In conclusion, the traditional surgical treatment aimed at correcting single predisposing factors based on the Lyonnaise "à la carte menu" approach led to satisfactory results in the treatment of OPI in our case series; however, long-term follow-up showed that this approach is not effective in preventing the occurrence of PF OA. Therefore, such patients have to be trained on correct attitudes and behaviors during working and sports activities to avoid facing any further risk of knee pain and occurrence of OA.

\section{Funding}

Research results reported here were partly supported by NSoSOT (Nuova Società Sarda di Ortopedia e Traumatologia) through the "Prof. F. Sotgiu" Award given during the 36th Regional Symposium.

\section{Conflict of Interest}

None.

\section{References}

1 Dejour D, Reynaud P, Lecoultre B. Douleurs et instabilité rotulienne. Essai de classification. Med Hyg (Geneve) 1998;56: 1466-1471

2 Dejour H, Walch G, Nove-Josserand L, Guier C. Factors of patellar instability: an anatomic radiographic study. Knee Surg Sports Traumatol Arthrosc 1994;2(01):19-26

3 Dejour DH. The patellofemoral joint and its historical roots: the Lyon School of Knee Surgery. Knee Surg Sports Traumatol Arthrosc 2013;21(07):1482-1494

4 Allain J, Dejour D. Arthrose fémoro-patellaire isolée. Symposium SOFCOT 2003. Rev Chir Orthop Reparatrice Apar Mot 2003; 90:109-119

5 Trillat A, Dejour H, Couette A. Diagnostic et traitement des subluxations recidivantes de la rotule. Rev Chir Orthop Repar Appar Mot 1964;50:813-824

6 Iwano T, Kurosawa H, Tokuyama H, Hoshikawa Y. Roentgenographic and clinical findings of patellofemoral osteoarthrosis. With special reference to its relationship to femorotibial osteoarthrosis and etiologic factors. Clin Orthop Relat Res 1990;(252): 190-197

7 Laurin CA, Lévesque HP, Dussault R, Labelle H, Peides JP. The abnormal lateral patellofemoral angle: a diagnostic roentgenographic sign of recurrent patellar subluxation. J Bone Joint Surg Am 1978;60(01):55-60

8 Roux C. Recurrent dislocation of the patella: operative treatment. Rev Chir Orthop Repar Appar Mot 1988;8:682

9 Brown DE, Alexander AH, Lichtman DM. The Elmslie-Trillat procedure: evaluation in patellar dislocation and subluxation. Am J Sports Med 1984;12(02):104-109

10 Kumar A, Jones S, Bickerstaff DR, Smith TW. Functional evaluation of the modified Elmslie-Trillat procedure for patello-femoral dysfunction. Knee 2001;8(04):287-292

11 Nakagawa K, Wada Y, Minamide M, Tsuchiya A, Moriya H. Deterioration of long-term clinical results after the Elmslie-Trillat procedure for dislocation of the patella. J Bone Joint Surg Br 2002; 84(06):861-864

12 Carney JR, Mologne TS, Muldoon M, Cox JS. Long-term evaluation of the Roux-Elmslie-Trillat procedure for patellar instability: a 26-year follow-up. Am J Sports Med 2005;33(08):12201223

13 Conti C, Berruto M, Bianchi M. The Elmslie-Trillat procedure for recurrent subluxation of the patella. One to five year follow-up. Ital J Orthop Traumatol 1992;18(03):341-349

14 Servien E, Verdonk PC, Neyret P. Tibial tuberosity transfer for episodic patellar dislocation. Sports Med Arthrosc Rev 2007;15 (02):61-67

15 Farr S, Huyer D, Sadoghi P, Kaipel M, Grill F, Ganger R. Prevalence of osteoarthritis and clinical results after the Elmslie-Trillat procedure: a retrospective long-term follow-up. Int Orthop 2014;38(01):61-66

16 Schüttler KF, Struewer J, Roessler PP, et al. Patellofemoral osteoarthritis after Insall's proximal realignment for recurrent patellar dislocation. Knee Surg Sports Traumatol Arthrosc 2014;22(11): 2623-2628

17 Sillanpää PJ, Mattila VM, Visuri T, Mäenpää H, Pihlajamäki H. Patellofemoral osteoarthritis in patients with operative treatment for patellar dislocation: a magnetic resonance-based analysis. Knee Surg Sports Traumatol Arthrosc 2011;19(02):230-235 